\title{
Watch one, do one, teach one
}

Marc Gillinov, MD, Stephanie Mick, MD, Tomislav Mihaljevic, MD, and Rakesh M. Suri, MD

From the Department of Thoracic and Cardiovascular Surgery, Cleveland Clinic, Cleveland, Ohio.

Disclosures: Authors have nothing to disclose with regard to commercial support.

Received for publication Feb 12, 2016; accepted for publication Feb 13, 2016; available ahead of print March 15, 2016.

Address for reprints: Marc Gillinov, MD, Cleveland Clinic/Desk J4-1, 9500 Euclid Ave, Cleveland, OH 44195 (E-mail: gillinom@ccf.org).

J Thorac Cardiovasc Surg 2016;151:1506-7

$0022-5223 / \$ 36.00$

Copyright (c) 2016 by The American Association for Thoracic Surgery

http://dx.doi.org/10.1016/j.jtcvs.2016.02.033

HOW CAN WE BEST TRAIN SURGICAL RESIDENTS TO PERFORM ROBOTIC SURGERY?

In this issue of the Journal, Valdis and colleagues ${ }^{1}$ report a randomized controlled trial that seeks to answer this question. ${ }^{1}$

In their study, Valdis and colleagues ${ }^{1}$ assigned 40 surgical trainees to 1 of 4 different training regimens: wet lab (porcine surgery), dry lab (skill drills), virtual reality (simulator), or control (no additional training). Subjects' skill levels with robotic surgery were tested before and after training, focusing on their ability to take down an internal thoracic artery and to place mitral annuloplasty sutures (Figure 1). Trainees who participated in the wet lab achieved the greatest improvement in skills, whereas those performing virtual reality robotic simulation also improved considerably. Dry lab training-basically the performance of drills with the robot-resulted in more modest gains.

\section{WHAT DO THESE RESULTS IMPLY?}

The first message is simple: Training is necessary before a surgeon can perform robotic cardiac surgery. At baseline, none of the 40 surgical trainees demonstrated expertise in robotic surgery. The dictum "Watch one then do one" does not apply here. Without specific, focused training on the robot, residents (and attending surgeons) cannot perform well in the operating room. By extending this finding, it is reasonable to conclude that surgeons who have not participated in a formal training program should not be permitted to perform robotic surgery on patients.

The second message generated by this study concerns the type of training. Wet lab training is the most effective at teaching particular skills and parts of a procedure. After only 2 hours of wet lab training, residents were able to achieve a preset level of proficiency at internal thoracic artery takedown and mitral annuloplasty. The superiority of wet lab training is due to the fact that the wet lab replicated the tasks on which the trainees were measured; in other words, practice in the wet lab was, in this case, "the real thing."

By recognizing the dramatic strides in virtual reality and imaging technology, one might have presumed that

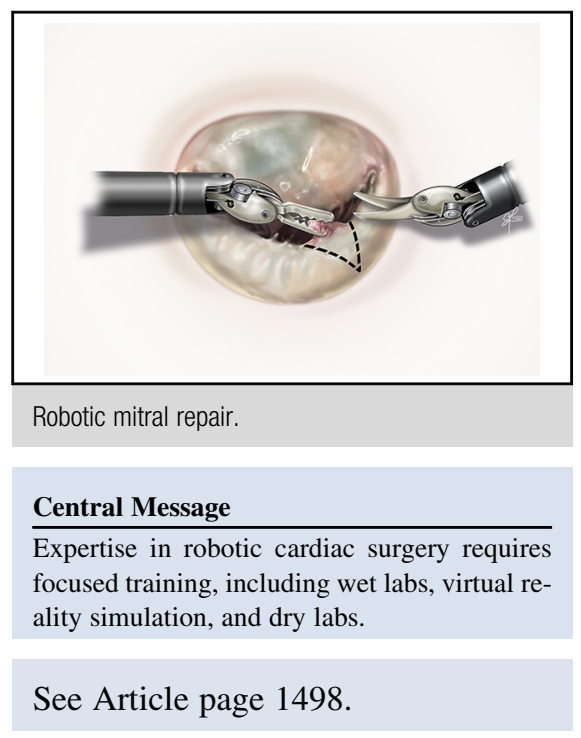

simulation training would equal wet lab training. Those assigned to the virtual reality simulation did become proficient, but the mean time to achieve this goal was approximately 5 times that required to achieve proficiency in the wet lab. Apparently, the simulation systems available are valuable but cannot yet substitute for actual work on tissue. Finally, those engaged in dry lab training achieved proficiency, but not to the level of those in the other groups. Dry lab training alone is inadequate preparation for surgery on patients.

\section{HOW SHOULD WE TRAIN SURGEONS FOR ROBOTIC SURGERY?}

No single training paradigm fits every resident and every institution. Given the demonstrated value of the 3 training strategies, it makes the most sense to use a combination of modalities. The program should be tailored to the institution's capabilities and the trainee's needs. At Cleveland Clinic, we use wet lab training, dry lab training, and simulation. Dry lab training comes first. By performing "skill drills," operators become familiar with robotic technology and its capabilities. We then move to formal wet lab training, generally providing 2 or 3 sessions. Simulation training is added after the wet lab training begins, and simulation is used periodically to continue to improve skills as the trainee begins to perform parts of actual operations.

\section{WHEN IS IT OKAY TO OPERATE ON A PERSON?}

The current study was not designed to answer this difficult question. Ultimately, this question can be answered only by the trainee and the attending/training surgeon. Patient safety may be jeopardized by premature introduction 


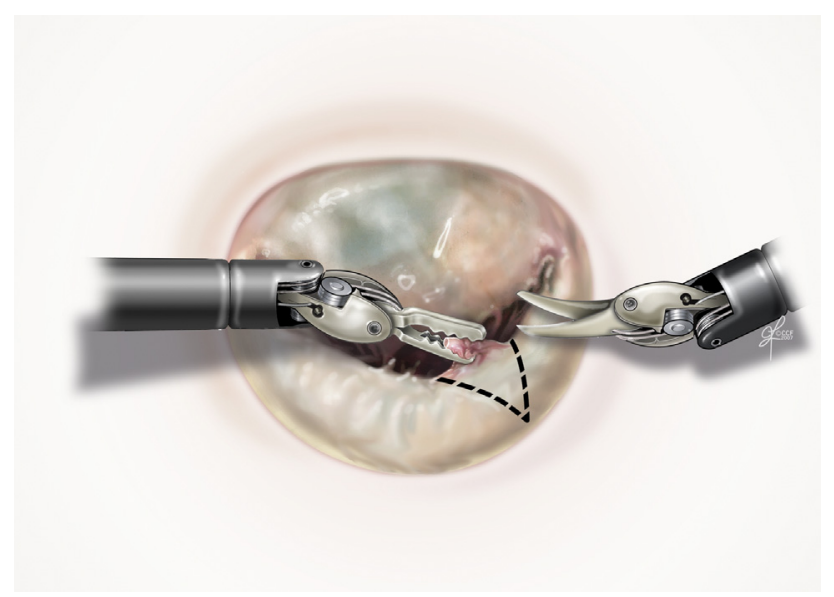

FIGURE 1. Robotic mitral valve repair: Triangular resection for posterior leaflet prolapse.

of robotic students to live patients when time-sensitive procedures are being performed (eg, those involving cardiopulmonary bypass and an arrested heart). Before participating in such an operation, the trainee should understand the robot's capabilities and be comfortable with its operation. It is wise to begin the experience by performing only components of the operation; over time, the trainee can perform a greater proportion of the case, eventually developing the capability to perform the entire operation. Finally, before going solo, the trainee should be proctored for several cases.

\section{PARADIGMS IN SURGICAL TRAINING: WHAT WOULD HALSTED SAY?}

Appointed surgeon-in-chief of the Johns Hopkins Hospital in 1890, William Stewart Halsted is credited with starting the first surgical residency program in the United States. Based on the traditional apprentice model, this system used "hands-on" training over a period of many years. With today's technology, this original approach must be augmented to achieve the best training in the most efficient manner. Endless hours observing surgery while holding retractors in the operating room does not constitute adequate training. Valdis and colleagues ${ }^{1}$ demonstrate that wet labs, dry labs, and virtual reality simulation can be used to develop skills in robotic surgery. Halsted would agree that we should use them all.

\section{Reference}

1. Valdis M, WA Chu M, Schlacchta C, Kiaii B. Evaluation of robotic cardiac surgery simulation training: a randomized controlled clinical trial. J Thorac Cardiovasc Surg. 2016;151:1498-505.e2. 